\title{
ON THE ITERATED LOGARITHM LAW FOR LOCAL TIME
}

\author{
EDWIN PERKINS
}

ABstract. If $s(t, x)$ is the local time of a Brownian motion, we show that

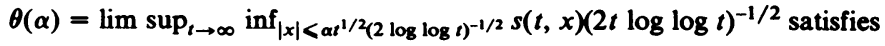

$$
\left(\left(1-\alpha^{1 / 2}\right) \vee 0\right)^{2}<\theta(\alpha)<(2 \alpha)^{-1} \wedge 1 .
$$

In particular, it follows from a result of Kesten that

$$
\limsup _{t \rightarrow \infty} s(t, x)(2 t \log \log t)^{-1 / 2}=1
$$

for all $x$ a.s.

1. Introduction. Suppose $B(t)$ is Brownian motion on a complete probability space $(\Omega, \mathcal{F}, P)$ and $s(t, x)=(d / d x) \int_{0}^{t} I(B(s) \leqslant x) d s(I(A)$ is the indicator function of $A)$ is its jointly continuous local time. Since $s(t, 0)$ is identical in law to $\sup _{s<t} B(s)$, the law of the iterated logarithm implies that lim $\sup _{t \rightarrow \infty} s(t, x) \phi(t)^{-1}$ $=1$ a.s. for each real $x$, where $\phi(t)=(2 t|\log | \log t||)^{1 / 2}$. In Kesten [1] it is shown that

$$
\underset{t \rightarrow \infty}{\lim \sup } \sup _{x \in \mathbf{R}} s(t, x) \phi(t)^{-1}=1 \text { a.s. }
$$

This implies that $\lim \sup _{t \rightarrow \infty} s(t, x) \phi(t)^{-1} \leqslant 1$ for all real $x$ a.s. but leaves open the question as to whether or not there is equality for all $x$ a.s. That there is equality for all $x$ a.s. is an easy corollary of the following theorem.

THEOREM 1. Let $\Psi(t)=t^{1 / 2}(2|\log | \log t||)^{-1 / 2}$. There is a nonincreasing function $\theta(\alpha)(\alpha \geqslant 0)$ such that

(a) lim $\sup _{t \rightarrow \infty} \inf _{|x| \leqslant \alpha \Psi(t)} s(t, x) \phi(t)^{-1}=\theta(\alpha)$ a.s. for all $\alpha \geqslant 0$,

(b) $\theta(\alpha) \leqslant(2 \alpha)^{-1} \wedge 1$ for all $\alpha \geqslant 0$,

(c) $\theta(\alpha) \geqslant\left(1-\alpha^{1 / 2}\right)^{2}$ for all $\alpha \leqslant 1$.

The method of proof is that in Kesten [1] but some simplification occurs due to the use of a maximal inequality for submartingales.

\section{Main result.}

Notation 2. If $a \geqslant 0$, let $T(a)=\inf \{t \geqslant 0 \mid s(t, 0)>a\}$.

LEMMA 3. If $a \geqslant 0, s(T(a), x)$ is a martingale in $x \geqslant 0$ and satisfies

$$
E\left(e^{-\lambda s(T(a), x)}\right)=\exp \left\{-\lambda a(1+2 \lambda|x|)^{-1}\right\} \quad(\lambda>0) .
$$

Proof. By Knight [2], $s(T(a), x)$ is a diffusion in $x \geqslant 0$ with infinitesimal generator $2 y d^{2} / d y^{2}$, and in particular is a nonnegative local martingale. Moreover,

Received by the editors March 7, 1980.

AMS (MOS) subject classifications (1970). Primary 60J55, $60 \mathrm{~J} 65$.

(C) 1981 American Mathematical Society 0002-9939/81/0000-0128/\$01.75 
(2) is derived in the proof of Corollary 1.2 in Knight [2]. It follows from (2) that $E(s(T(a), x))=a<\infty$ for all $x$ and hence $s(T(a), x)$ is a supermartingale by Fatou's lemma. Since $E(s(T(a), x))$ is independent of $x$, we see that $s(T(a), x)$ must in fact be a martingale.

Proof of Theorem 1. Since lim $\sup _{t \rightarrow \infty} \inf _{|x|<\alpha \Psi(t)} s(t, x) \phi(t)^{-1}$ is measurable with respect to the tail $\sigma$-field of a Brownian motion, a well-known zero-one law implies that the above expression is a.s. equal to a nonnegative constant $\theta(\alpha)$. Clearly $\theta(\alpha) \leqslant 1$ because $\lim \sup _{t \rightarrow \infty} s(t, 0) \phi(t)^{-1}=1$ a.s. Moreover if $t>e$, then

$$
\begin{aligned}
\inf _{|x|<\alpha \Psi(t)} s(t, x) \phi(t)^{-1} & \leqslant \int_{-\alpha \Psi(t)}^{\alpha \Psi(t)} s(t, x) \phi(t)^{-1} d x(2 \alpha \Psi(t))^{-1} \\
& <t(2 \alpha \phi(t) \Psi(t))^{-1}=(2 \alpha)^{-1}
\end{aligned}
$$

It remains to show (c). Fix $\alpha \in[0,1)$ and $\alpha_{1} \in\left(0,\left(1-\alpha^{1 / 2}\right)^{2}\right)$. Then choose $\alpha_{2} \in\left(\alpha_{1} \vee \alpha, 1\right)$ such that $\alpha_{1}<\left(\alpha_{2}^{1 / 2}-\alpha^{1 / 2}\right)^{2}$ or, equivalently, $\alpha<$ $\left(\alpha_{2}^{1 / 2}-\alpha_{1}^{1 / 2}\right)^{2}$. The usual proof of the law of the iterated logarithm allows us to choose $t>1$ such that $P\left(T_{k}<t^{k}\right.$ infinitely often $)=1$, where $T_{k}=T\left(\alpha_{2} \phi\left(t^{k}\right)\right)$. Therefore

$$
\begin{aligned}
P\left(\inf _{|x|<\alpha \Psi\left(T_{k}\right)} s\left(T_{k}, x\right) \phi\left(T_{k}\right)^{-1}>\alpha_{1} \text { infinitely often }\right) \\
\quad \geqslant P\left(\inf _{|x|<\alpha \Psi\left(t^{k}\right)} s\left(T_{k}, x\right)>\alpha_{1} \phi\left(t^{k}\right) \text { and } T_{k}<t^{k} \text { infinitely often }\right) \\
\quad \geqslant P\left(\sup _{|x|<\alpha \Psi\left(t^{k}\right)} s\left(T_{k}, 0\right)-s\left(T_{k}, x\right) \geqslant\left(\alpha_{2}-\alpha_{1}\right) \phi\left(t^{k}\right) \text { only finitely often }\right) .
\end{aligned}
$$

Use a maximal inequality for submartingales and Lemma 3 to see that if $\lambda>0$, then

$$
\begin{aligned}
& P\left(\sup _{|x|<\alpha \Psi\left(t^{k}\right)} s\left(T_{k}, 0\right)-s\left(T_{k}, x\right)>\left(\alpha_{2}-\alpha_{1}\right) \phi\left(t^{k}\right)\right) \\
& \quad \leqslant 2 P\left(\sup _{0<x<\alpha \Psi\left(t^{k}\right)} s\left(T_{k}, 0\right)-s\left(T_{k}, x\right)>\left(\alpha_{2}-\alpha_{1}\right) \phi\left(t^{k}\right)\right) \\
& \quad \leqslant 2 \exp \left\{-\lambda\left(\alpha_{2}-\alpha_{1}\right) \phi\left(t^{k}\right)\right\} E\left(\exp \left\{\lambda\left(s\left(T_{k}, 0\right)-s\left(T_{k}, \alpha \Psi\left(t^{k}\right)\right)\right)\right\}\right) \\
& \quad=2 \exp \left\{-\lambda\left(\alpha_{2}-\alpha_{1}\right) \phi\left(t^{k}\right)+\lambda \alpha_{2} \phi\left(t^{k}\right)-\lambda \alpha_{2} \phi\left(t^{k}\right)\left(1+2 \lambda \alpha \Psi\left(t^{k}\right)\right)^{-1}\right\} \\
& \quad=2 \exp \left\{-\lambda \phi\left(t^{k}\right)\left(\alpha_{2}\left(1+2 \lambda \alpha \Psi\left(t^{k}\right)\right)^{-1}-\alpha_{1}\right)\right\} .
\end{aligned}
$$

An elementary calculus argument shows that (4) has a minimum value of

$$
2 \exp \left\{-\left(\alpha_{2}^{1 / 2}-\alpha_{1}^{1 / 2}\right)^{2} \alpha^{-1}|\log | \log t^{k}||\right\}
$$

when $\lambda=\left(\left(\alpha_{2} \alpha_{1}^{-1}\right)^{1 / 2}-1\right)\left(2 \alpha \Psi\left(\mathrm{t}^{\mathrm{k}}\right)\right)^{-1}$. Since $\left(\alpha_{2}^{1 / 2}-\alpha_{1}^{1 / 2}\right)^{2}>\alpha$, (5) is summable over $k$ and therefore

$$
P\left(\sup _{|x|<\alpha \Psi\left(t^{k}\right)} s\left(T_{k}, 0\right)-s\left(\dot{T}_{k}, x\right) \geqslant\left(\alpha_{2}-\alpha_{1}\right) \phi\left(t^{k}\right) \text { only finitely often }\right)=1
$$


by the Borel-Cantelli lemma. It follows from (3) that

$$
\limsup _{k \rightarrow \infty} \inf _{|x|<\alpha \Psi\left(T_{k}\right)} s\left(T_{k}, x\right) \phi\left(T_{k}\right)^{-1} \geqslant \alpha_{1} \text { a.s. }
$$

for all $\alpha_{1}<\left(1-\alpha^{1 / 2}\right)^{2}$, and hence $\theta(\alpha) \geqslant\left(1-\alpha^{1 / 2}\right)^{2}$.

Since $\theta(\infty)=0$, if $h(t)$ satisfies $\lim _{t \rightarrow \infty} h(t) \Psi(t)^{-1}=+\infty$ then

$$
\limsup _{t \rightarrow \infty} \inf _{|x|<h(t)} s(t, x) \phi(t)^{-1}=0 \text { a.s., }
$$

and since $\theta\left(0^{+}\right)=1$, if $h(t)$ satisfies $\lim _{t \rightarrow \infty} h(t) \psi(t)^{-1}=0$ then

$$
\limsup _{t \rightarrow \infty} \inf _{|x|<h(t)} s(t, x) \phi(t)^{-1}=1 \text { a.s. }
$$

This latter result (with $\lim _{t \rightarrow \infty} h(t)=\infty$ ), coupled with (1), gives us the following corollary.

Corollary 4. For $\omega$ outside a single null set, $\lim \sup _{t \rightarrow \infty} s(t, x) \phi(t)^{-1}=1$ for all $x$.

REMARK 5. A trivial modification of the proof of Theorem 1 shows that for all $\alpha \geqslant 0$ there is a constant $\theta^{1}(\alpha)$ satisfying (b) and (c) of Theorem 1 and also

$$
\limsup _{t \rightarrow 0^{+}} \inf _{|x|<\alpha t^{1 / 2}\left(2 \log \log t^{-1}\right)^{-1 / 2}} s(t, x)\left(2 t \log \log \left(t^{-1}\right)\right)^{-1 / 2}=\theta^{1}(\alpha) \quad \text { a.s. }
$$

Acknowledgement. I would like to thank Professor O. Stackelberg for suggesting this problem.

\section{REFERENCES}

1. H. Kesten, An iterated logarithm law for local time, Duke Math. J. 32 (1965), 447-456.

2. F. B. Knight, Random walks and a sojourn density process of Brownian motion, Trans. Amer. Math. Soc. 109 (1963), 56-86.

Department of Mathematics, Universtty of BrTtish Columbia, Vancouver, British Columbia, Canada V6T 1W5 